\title{
The Research on Pinning Control of Chinese Aviation Network based on Robustness analysis
}

\author{
Yao Hongguang \\ College of Aviation Transportation \\ Shanghai University of Engineering and Science \\ ShangHai, China \\ yhg1yhg@sina.com
}

\author{
Li Zhizhong \\ College of Aviation Transportation \\ Shanghai University of Engineering and Science \\ ShangHai, China \\ yhg1yhg@sina.com
}

\begin{abstract}
If some nodes of the aviation network are pinning controlled, all nodes are synchronized to the steady state and promote aviation network robustness. Collecting the data information of routes, China aviation network's statistical characteristic is calculated, and aviation network robustness metrics is put forward; through the establishment of state equation of the node, determine the aviation network equilibrium, realize to the partial node pinning control, and controlled the number of nodes and the feedback gain overall cost minimum standards, exploring both balanced relationship. The results show that, based on 45 navigation city of pinning control, can lower feedback gain level, realize Chinese aviation network robustness improvement.
\end{abstract}

Keywords- aviation network; pinning control; robustness analysis; complex networks; simulation

Not: This paper is funded by the 2013 Shanghai education committee key project of scientific research innovation(13ZS127), 2014 the ministry of education of humanities and social science research youth fund projects (14YJCZH183).

\section{INTRODUCTION}

Air transport is easily affected by external interference, so the robustness of the study to improve the whole network reliability of aviation, air transport to ensure the safe and efficient operation of significance, more and more scholars attention. Zhang Wenwen presents a simulation analysis method for the analysis of aviation network robust methods, through research the aviation network in response to random attack has high robustness to attacks is more fragile ${ }^{[1]}$. Deng Gui Shi application of complex network technology, based on the minimum total cost of the network optimization mathematical model proposed route robustness determination method, and makes the empirical research ${ }^{[2]}$. What spring is systematically discusses the complex network structure on robustness effect ${ }^{[3-4]}$. At present, the domestic and the robustness of the complex network research mainly on robustness for the quantitative analysis of complex network, but robust pinning control has also aroused extensive interest of researchers. For example, Wang proposed a effective method to make the network all nodes are pinning control to its equilibrium point ${ }^{[5-6]}$;
Chen using a node to contain and control the whole network ${ }^{[7]}$; Zhou using adaptive method to contain the entire network ${ }^{[8]}$. Zhao military products, made through the establishment of cost function reaches its minimum value, in order to achieve a controlled number of nodes and the feedback gain is a kind of balanced algorithm ${ }^{[9]}$.

\section{CHINA AVIATION NETWORK ROBUSTNESS ANALYSIS}

\section{A. Data collection}

Using data from the "flight Ctrip Travel Network" schedule, collected a total of 163 navigable City, 2198 domestic routes, flight 9611 . By taking 163 navigable city as a matrix of rows and columns, with flights between two city as the weight of the adjacency matrix of a weighted aviation network, A forms a $163 \times 163$.

\section{B. The aviation network robustness metrics}

In general, " the maximal connected sub-graphs relative to the size of" most metric aviation network robustness index. Its computational methods such as formula (1).

the number of nodeof the maximal connectedsubgraphs the number of nodeof network

" the maximal connected sub-graphs" contains the node number increasing representation of network connectivity is better, and when it is equal to the number of nodes in the network, all nodes that are interconnected. With a paralyzed nodes to increase in value, value will continue to change. The value of a certain, larger values, show on the network connectivity robustness.

\section{C. pinning control on improving aviation network robustness analysis of meaning}

Improving aviation network robustness, contribute to the discovery of aviation the important node in the network and effective protection, avoid suffer outside interference caused by delays and even lead to network paralysis, air transport to ensure the safe and efficient operation. Pinning control, in recent years has been widely applied to complex network dynamics and control, its basic idea is to only one part in network node directly 
exerts a constant input control, and achieves the effective suppression of the entire network spatiotemporal chaotic behavior. The airline network pinning control may be implemented in a network robustness improvement goals at the same time, greatly reduce the number of nodes to reduce the controlled, control cost has important sense.

\section{CHINESE AVIATION NETWORK PINNING CONTROL RESEARCH}

\section{A. The network node of linearly coupled ordinary differential equations}

Linearly coupled ordinary differential equations are used to describe the continuous dynamical systems are important tools [11]. In general, it can be described as the following form:

$$
\dot{X}_{i}=f\left(x_{i}\right)+c \sum_{j=1}^{N} c_{i j} x_{j}
$$

In which: $N>1$ :network number; $\mathrm{f}$ : is a continuous function; $\mathcal{X}_{i}$ : the i node state variables; $c>0$ is the network of the coupling strength; $C=\left(c_{i j}\right)_{N \times N}$ : coupling matrix, reaction network topology in which if the node $\mathrm{i}$ and node $\mathrm{j}\left({ }^{i \neq j}\right)$ there is a connection, then $c_{i j}=1$, otherwise $c_{i j}=0$, C need not be symmetric, but its content formula (3), which $k_{i}$ for the node i degree.

$$
c_{i i}=-\sum_{j=1, j \neq i}^{n} c_{i j}=-k_{i}
$$

\section{B. controlled node network is described}

The implementation of network synchronization when the $\mathrm{x}$ value as a control balance:

$$
x_{1}=x_{2}=\cdots=x_{N}=\bar{x}, \text { and } f(\bar{x})=0
$$

In order to aviation network control to the equilibrium point, need to account for the proportion of the total number of network nodes for a small portion of the nodes $\sigma(0<\sigma<1)$ involved control implementation. If nodes $\boldsymbol{i}_{1}, \boldsymbol{i}_{2}, \cdots, \boldsymbol{i}_{r}$ are selected as being involved in the control of the node, $r$ expressed by the number of nodes involved control. In the linear feedback function, node to contain the network can be described as follows:

$$
\begin{aligned}
& \dot{\mathcal{X}}_{i_{k}}=f\left(\boldsymbol{X}_{i_{k}}\right)+c\left(\sum_{j=1_{k}}^{N} \boldsymbol{C}_{i_{k}} \boldsymbol{X}_{j}-d \boldsymbol{e}_{i_{k}}\left(\boldsymbol{X}_{i_{k}}-\bar{x}\right)\right) \quad, \quad 1 \leq i \leq r \\
& \dot{\mathcal{X}}_{i_{k}}=f\left(\boldsymbol{X}_{i_{k}}\right)+c \sum_{j=1_{k}}^{N} \boldsymbol{C}_{i_{k} j} \boldsymbol{X}_{j} \quad, \quad i>r
\end{aligned}
$$

Wherein: ${ }^{e_{i_{k}}}$ said controlled node in achieving normal airport functions of the price, namely the airport facilities a total investment and operation cost; $d>0$ is the feedback control gain coefficient, $d e_{i_{k}}$ said feedback gain. In the aviation network, feedback control gain practical significance is: each node in the" airport meteorological factors"," flow surge"," facility fault" and other unexpected effects when they occur, can still maintain the normal function and do extra pay. This additional preparation includes not only the airport in terms of hardware, infrastructure upgrades, improve and reserve, including management, process optimization, because the hardware investment and operation cost is huge, so the extra cost is great.

\section{C. controlled node number $R$ and feedback control gain equalization}

In the traditional pinning control study, in order to make the power network stability to a predetermined point, usually the feedback control gain coefficient $d$ is set to be large enough; however, in the aviation network control of $d$ value high enough is clearly not feasible; when $d=1$, it means to cope with the unexpected situation while the extra cost and the entire airport investment and operating costs and the same, in reality it is impossible; only the $d$ value control in a sufficiently small level of aviation network, pinning control have practical meaning.

If for $\varepsilon_{0}>0$, there is a natural number $r \in[1, N-1]$, and a feedback gain factor $d$ meet formula (5), by pinning network formula (4) can be stabilized to the equilibrium point $^{[10]}$.

$$
\lambda_{\text {max }}\left(\left(\hat{C}+\hat{C}^{T}\right) / 2-D / \lambda_{\max }(P)\right)<\lambda_{r+1}+\varepsilon_{0}<-\sigma / c
$$

Whichin: $\lambda_{i}$ is $M_{i}$ the largest eigenvalue of matrix $\left(\hat{C}+\hat{C}^{T}\right) / 2 ; M_{i}$ is a matrix $\left(\hat{C}+\hat{C}^{T}\right) / 2$ emoval of $1,2, \cdots, N-1$ rows and columns of the matrix, and $\hat{C}$ is matrix which main diagonal elements $c_{i i}$ being replaced by $\quad\left[\lambda_{\min }(p) / \lambda_{\max }(p)\right]_{C_{i i}}$ $D=\left(\begin{array}{cc}D_{1} & 0 \\ 0 & 0\end{array}\right), D_{1}=\operatorname{diag}\left(d_{1}, \cdots, d_{n}\right)$ is a positive definite $\operatorname{matrix}^{[11]}$.

There is the assumption which $\hat{\lambda}_{1}$ is the largest eigenvalue of matrix $\left(\hat{C}+\hat{C}^{T}\right) / 2-D / \lambda_{\max }(P)$, if so $\hat{\lambda}_{1}=\lambda_{r+1}$, then the feedback gain to achieve $d$ value is large enough, this is not practical; therefore, the introduction of a relaxation factor $\varepsilon_{0}$, makes for an appropriate $d, \hat{\lambda}_{1} \leq \lambda_{r+1}+\varepsilon_{0}$ set up. Check node more, meet the above inequality for the feedback gain of $d$ smaller ${ }^{[12]}$. For a given pinned node number $r$, the feedback gain factor $d$ of the existence of a critical value, when $d$ is greater than the critical value, the aviation network controlled node contain network can reach a stable equilibrium point. Similarly, for a given feedback gain of $d$, contain a number of nodes is the existence of a critical value, amounting to less than the value, controlled node contain network would not reach the exponential stability of the state. Therefore, we can get a series of feasible combinations ( $r, d)$, which defines a stable region, it can be used to divide the check node number $r$ and feedback gain of $d$ stable parameter space ${ }^{[15]}$. 
A cost function is $Q(r, d)=r^{\alpha} d^{1-\alpha}$, can be obtained minimum( $r, d$ )feasible combination, thus combining the two value.

\section{CHINESE AVIATION NETWORK PINNING CONTROL EMPIRICAL ANALYSIS}

\section{A. Chinese aviation network pinning control}

From the aviation network robustness analysis point of view, the state nodes airport includes 2 kinds : normal state, i.e. for all flights to provide normal transport services; state of paralysis, which can provide normal transport service, or business hours flight waiting too long.

The flight at the airport, waiting for the standard business hours, using queuing theory, it can be Chinese aviation network node of linearly coupled ordinary differential equations:

$$
\left[\begin{array}{l}
\dot{X}_{i_{1}} \\
\dot{x}_{i_{2}}
\end{array}\right]=\left[\begin{array}{c}
\frac{z_{i}}{\boldsymbol{x}_{i}}+c \sum_{j=1}^{N} c_{i j} x_{j} \\
\frac{z_{i}{ }^{2}}{x_{i}-z_{i}}
\end{array}\right] i=1,2, \cdots, N
$$

Wherein: $\dot{X}_{i_{1}}$ said node airport paralyzed; $\dot{X}_{i_{2}}$ said node airport in the normal state; $N>1$ : aviation network node city number, as of May 16, 2011, China has fixed flight navigation city a total of $163 ; z_{i}$ said that the $i$ node airport normal unit time aviation mean flow, from" see civil aviaton from statistic - Airport vehicles and passenger throughput statistics" get the relevant data; $x_{i}$ : said that the $i$ node airport when receiving external interference after the unit of time service flights; $c>0$ is aviation network coupling strength, each node city affect the relationship between the nodes in the network, can use air city" degree" of the average value to express Chinese aviation network, each node degree average $c=13.736$; $C=\left(c_{i j}\right)_{N \times N}$ is coupling matrix, reaction of aviation network topology, which has collected $163 \times 163$ of the airline network adjacency matrix $C$.

The realization of node airport normal function of control point of equilibrium, according to equation (5) to establish Chinese aviation network node to contain the network equations. And the use of literature ${ }^{[8]}$ : Zhao military products and other scholars to" determine network minimum coupling strength and minimal pinned node, increasing pinned node, the final selection of a suitable, so that the cost function reaches its minimum value, then get the best $(r, d)$ combination " algorithm, computation gives Chinese aviation network controlled points and feedback gain coefficients possible combinations $(r, d)$ as shown in table 1 .
TABLE I. FEASIBLE COMBINATION TABLE

\begin{tabular}{|c|c|c|c|c|c|c|c|}
\hline & 1 & 2 & 3 & 4 & 5 & 6 & 7 \\
\hline$r$ & 25 & 29 & 33 & 38 & 42 & 45 & 50 \\
\hline$d$ & 52.4 & 38.3 & 20.4 & 19.8 & 10.5 & 9.6 & 8.9 \\
\hline
\end{tabular}

When $\alpha$ in $[0.1]$ between with different values, the feasible combinations of worth changes as shown in figure 1 .

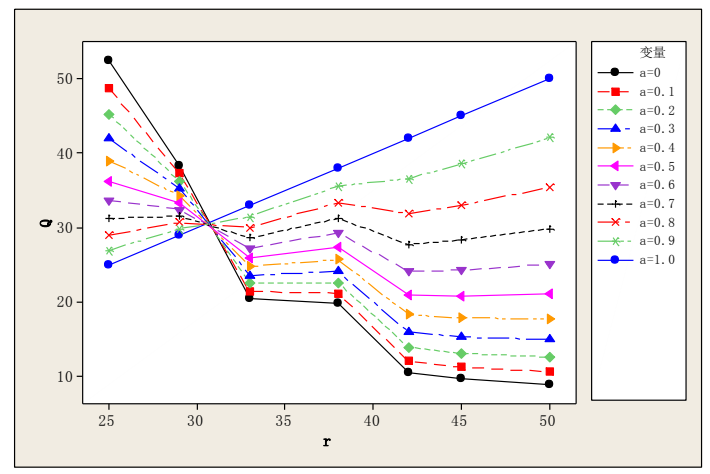

Figure 1. feasible combinations of the corresponding $\mathrm{Q}$ value

Through calculation, when at the same time both $r, d$ value, this time when $r=45, Q$ has minimum value. For Chinese aviation network, as long as the choice of control 45 key nodes, we will be able to reserve the controlled node $9.6 \%$ service capacity for the price, to achieve effective control of the entire network.

\section{B. 45 controlled node analysis}

Because the Chinese aviation network node of linearly coupled ordinary differential equations, in order to" degree" of the mean value to reflect the aviation network coupling strength, therefore from the aviation network robustness effect point of view, should choose Ranking in the top 45 of the city as a pinning control object.

In the network, the node degree and node is defined as the total number of border. In the aviation network, a navigable city of greater means connected with the city number, it has few routes are also more. Application of large scale network analysis software Ucinet ( version 6.212 ), and collected $163 \times 163$ of the China aviation network adjacency matrix $C$, calculate the Chinese 163 navigable city degree, the top 45 navigation city as shown in table 2. The 45 node city has a total of 1767 routes, routes in total quantity $80.39 \%$, formed a Chinese aviation network core.

TABLE II. THE TOP 45 NAVIGABLE CITY VALUE TABLE

\begin{tabular}{|c|c|c|}
\hline $\begin{array}{c}\text { order of } \\
\text { degree }\end{array}$ & degree & city \\
\hline 1 & 118 & BJS \\
\hline 2 & 95 & CAN \\
\hline 3 & 95 & SHA \\
\hline 4 & 68 & CTU \\
\hline 5 & 67 & KMG \\
\hline 6 & 66 & SZX \\
\hline 7 & 62 & SIA \\
\hline 8 & 62 & CKG \\
\hline 9 & 52 & XMN \\
\hline
\end{tabular}




\begin{tabular}{|c|c|c|}
\hline 10 & 46 & CSX \\
\hline 11 & 43 & DLX \\
\hline 12 & 43 & NKG \\
\hline 13 & 41 & HGH \\
\hline 14 & 40 & TAO \\
\hline 15 & 40 & TNA \\
\hline 16 & 40 & WUH \\
\hline 17 & 39 & KWE \\
\hline 18 & 39 & URC \\
\hline 19 & 37 & SHE \\
\hline 20 & 36 & TSN \\
\hline 21 & 36 & CGO \\
\hline 22 & 35 & HET \\
\hline 23 & 35 & WNZ \\
\hline 24 & 35 & HRB \\
\hline 25 & 34 & FOC \\
\hline 26 & 34 & NNG \\
\hline 27 & 33 & HAK \\
\hline 28 & 33 & SYX \\
\hline 29 & 32 & TYN \\
\hline 30 & 28 & KWL \\
\hline 31 & 28 & HFE \\
\hline 32 & 27 & INC \\
\hline 33 & 25 & NGB \\
\hline 34 & 25 & KHN \\
\hline 35 & 25 & SJW \\
\hline 36 & 24 & LHW \\
\hline 37 & 23 & CGQ \\
\hline 38 & 21 & JJN \\
\hline 39 & 19 & SWA \\
\hline 40 & 18 & YNT \\
\hline 41 & 14 & MIG \\
\hline 42 & 14 & XNN \\
\hline 43 & 14 & CZX \\
\hline 44 & 13 & ZUH \\
\hline 45 & 13 & XUZ \\
\hline
\end{tabular}

\section{CONCLUSION}

This paper establishes China route network node state of ordinary differential equations, and by pinning control method the optimization controlled node number and feedback gain. Through the research, the following conclusions are obtained:

(1) improving aviation network robustness, for ensuring safe and efficient air transport has important significance. Pinning control compared to other controls can be in the price under the same conditions, to ensure aviation network high robustness.

(2) according to the existing Chinese aviation network, with $9.6 \%$ feedback gain, to Beijing, Guangzhou, Shanghai 45 city are pinning control, are both controlled the number of nodes and the feedback gain is relatively reasonable scheme.

\section{ACKNOWLEDGMENT}

This paper is funded by the 2013 Shanghai education committee key project of scientific research innovation(13ZS127), 2014 the ministry of education of humanities and social science research youth fund projects (14YJCZH183).

\section{REFERENCES}

[1] Deng Gui-shi, Wu Pei-jian, Tian Wei .Research on robustness and vulnerability of global shipping network[J]. Journal of Dalian University of Technology, 2008, 48 (5) : 765-768.

2] Zhang Wenwen. Research on Application of Complex Network Theory in Aviation Network[D]. Civil Aviation University of China,2009.

[3] Chunhua Gao. Airline Integrated Planning and Operations [D].Georgia Institute of Technology, 2007.

[4] Wang X E, Chen C. Synchronization in scale-free dynamical networks: robustness and fragility[C]. IEEE trans Circ Syst-I, 2002(49): 54-62.

[5] Zhou J, Chen T. Synchronization in general complex delayed dynamical networks[C]. IEEE trans Circ Syst-I, 2006(53): 733-744.

[6] Porta.S, Crucitti.P, Latora.V. The network analysis of urban streets: A dual approach[J]. Physica A, 2006(369): 853-866.

[7] Guimero.R , Mossa.S , Turtschi.A. The world-wide air transportation network: Anomalous centrality, community structure, and cities'global roles[C]. Proc Natlacad SCI USA, 2007, 102(22): 7794-7799.

[8] Zhao Junchan, Lu junan, Wu xiaoqun. General complex dynamica networks with optimal pinning control[J].Scientia Sinica(Informationis),2010,(6):821-830.

[9] Chunhua Gao. Airline Integrated Planning and Operations [D].Georgia Institute of Technology, 2007.

[10] Wang X E, Chen C. Synchronization in scale-free dynamical networks: robustness and fragility[C]. IEEE trans Circ Syst-I, 2002(49): 54-62.

[11] Zhou J, Chen T. Synchronization in general complex delayed dynamical networks[C]. IEEE trans Circ Syst-I, 2006(53): 733-744.

[12] Porta.S, Crucitti.P, Latora.V. The network analysis of urban streets: A dual approach[J]. Physica A, 2006(369): 853-866.

[13] Guimero.R , Mossa.S , Turtschi.A. The world-wide air transportation network: Anomalous centrality, community structure, and cities'global roles[C]. Proc Natlacad SCI USA, 2007, 102(22): 7794-7799. 